\title{
High-risk human papillomavirus E6/E7 mRNA and L1 DNA as markers of residual/recurrent cervical intraepithelial neoplasia
}

\author{
MARIA PERSSON ${ }^{1}$, SOPHIA BRISMAR WENDEL ${ }^{1}$, LINDA LJUNGBLAD ${ }^{1}$, \\ BO JOHANSSON $^{2}$, ELISABETE WEIDERPASS ${ }^{3,4,5}$ and SONIA ANDERSSON ${ }^{1}$
}

\begin{abstract}
${ }^{1}$ Department of Women's and Children's Health, Division of Obstetrics and Gynecology, Karolinska Institutet, Elevhemmet H2:00, Karolinska University Hospital Solna, 17176 Stockholm; ${ }^{2}$ Department of Laboratory Medicine, Division of Clinical Virology, Karolinska Institutet, Karolinska University Hospital Huddinge, 14186 Stockholm; ${ }^{3}$ Department of Medical Epidemiology and Biostatistics Karolinska Institutet, 17177 Stockholm, Sweden; ${ }^{4}$ Cancer Registry of Norway, Postboks 5313, Majorstuen, 0304, Oslo and Department of Community Medicine, Universitetet I Tromso, 9037 Tromso, Norway; ${ }^{5}$ Samfundet Folkhälsan, Genetic Epidemiology Group, Folkhälsan Research Center, Biomedicum 1, University of Helsinki, 00014 Helsinki, Finland
\end{abstract}

Received February 10, 2012; Accepted March 14, 2012

DOI: $10.3892 /$ or.2012.1755

\begin{abstract}
The aim of this study was to assess the use of human papillomavirus (HPV) E6/E7 mRNA testing in the follow-up of women treated for cervical intraepithelial neoplasia (CIN) by conization and to compare the prognostic value of HPV E6/E7 mRNA to HPV L1 DNA and cytology. One hundred and forty-three women underwent cytological/histological testing, HPV DNA genotyping by Linear Array, and HPV E6/ E7 mRNA testing by APTIMA HPV assay during followup after surgical treatment for histologically verified CIN. High-grade residual/recurrent disease $\left(\mathrm{CIN} 2^{+} / \mathrm{HSIL}^{+}\right)$was identified in 7 (4.9\%) women, and low-grade disease (CIN1/ LSIL) in 25 (17.5\%). At the inclusion visit 33 (23\%) women were HPV DNA-positive; 13 (9.0\%) were HPV E6/E7 mRNApositive. HPV E6/E7 mRNA did not identify three women with high-grade disease. Presence of high-risk HPV DNA at the inclusion visit predicted $100 \%$ (95\% CI 64.6-100) of high-grade residual/recurrent disease, with a specificity of $80.9 \%$ (95\% CI 73.5-86.6); cytology had a sensitivity of $85.7 \%$, and a specificity of $87.5 \%$. HPV E6/E7 mRNA testing was a poor predictor of treatment failure, with a sensitivity of $57.1 \%$ (95\% CI 25.0-84.2), but high specificity (93.4\%; 95\% CI
\end{abstract}

Correspondence to: Dr Sonia Andersson, Department of Women's and Children's Health, Division of Obstetrics and Gynecology, Karolinska Institutet, Elevhemmet H2:00, Karolinska University Hospital Solna, 17176 Stockholm, Sweden

E-mail: sonia.andersson@karolinska.se

Key words: CIN, conization, genotyping, human papillomavirus, residual, recurrence, E6/E7 mRNA
87.9-96.5). Detection of high-risk HPV DNA after treatment by conization identified $100 \%$ of women with residual/recurrent high-grade disease, whereas HPV E6/E7 mRNA testing was a poor predictor of treatment failure. This study suggests that a negative HPV mRNA result cannot exclude the risk of malignant progression, and that HPV E6/E7 mRNA testing by APTIMA HPV assay is not useful in the follow-up of women treated for CIN.

\section{Introduction}

Cervical cancer is the third most common malignancy among women worldwide. Approximately 530,000 women develop cervical cancer and 275,000 die from it every year (1). The introduction of cytology-based screening programs has resulted in a significant decrease in the incidence and mortality rates of cervical cancer (2). Indeed, the disease is highly preventable as it is preceded by a well-recognized premalignant stage that can be identified by cytological/histological examination and treated (3). The two most common histological types of cervical cancer are squamous cell carcinoma (SCC) and adenocarcinoma.

High-risk (HR) human papillomavirus (HPV) is present in over $99 \%$ of analyzed SCC, and in most precancerous lesions (4). However, HR HPV infection is very common, and is transient in most women; only an estimated $10 \%$ of those with an HR HPV infection are subject to persistent infection, which is believed to cause SCC through expression of the viral oncoproteins E6 and E7 (5). These oncoproteins play a significant role in malignant transformation, and are consistently expressed in malignant tissue. Their mechanism of action is centered on the inactivation of the tumor suppressor proteins p53 and pRb $(6,7)$.

Increasing levels of HPV E6/E7 mRNA cause genetic instability, and imply a risk of cellular changes, resulting in 
a selective growth advantage $(8,9)$. Therefore, the presence of viral HPV E6/E7 mRNA transcripts could identify women at risk for residual/recurrent cervical intraepithelial neoplasia (CIN) with higher sensitivity and specificity than HPV DNA detection $(10,11)$. In screening programs, detection of fulllength E6/E7 mRNA of a number of HR HPV types has been shown to be highly associated with high-grade squamous intraepithelial lesions (HSIL) and SCC than HR HPV DNA detection (12-14).

Women who have been treated for CIN are considered to be at high-risk of developing invasive cervical cancer for many years after treatment, necessitating a long-term followup strategy to detect residual/recurrent CIN. Compared to cytology, HPV DNA testing allows quicker identification of residual/recurrent CIN (15-18), and has high sensitivity and negative predictive value (NPV) (18).

HPV E6/E7 mRNA is notably expressed in transforming infected squamous epithelium. Although little is known about its possible transient nature, it should be clinically interesting to monitor this expression after CIN treatment.

The aim of the present study was to assess the E6/E7 mRNA expression of 14 HR HPV types among women treated for CIN, to correlate HPV E6/E7 mRNA with subsequent cytological and histological results and to compare the prognostic value of HPV E6/E7 mRNA and HPV DNA testing.

\section{Materials and methods}

Patient selection. Women diagnosed with CIN in the regular cervical cancer screening program in Stockholm County are referred to the Department of Gynecology and Obstetrics, Karolinska University Hospital Huddinge (Karolinska), Stockholm, Sweden (a tertiary health care service) for treatment by conization. Karolinska also serves as a primary cervical cancer screening center for women residing in the surrounding area. The present study considered women treated for CIN by conization between September 1999 and June 2009 and of all treated women 149 returned for at least one follow-up visit and were invited to participate in the present study. In 2003, HPV L1 DNA testing became available at the Virology Department of at Karolinska, and the present study protocol was implemented (18). The 'inclusion visit' was defined as the first follow-up visit after conization. The study protocol was approved by the Karolinska Ethics Committee and all participants signed an informed consent form.

Treatment procedure and histological results. Of the 149 study women, 143 were treated by loop excision electrosurgical procedure (LEEP) using a C-LETZ electrode (Utah Medical Products Inc., Midvale, UT, USA) (19) between 1999 and 2009. Of the 6 study women who did not undergo LEEP, 2 were treated with cryotherapy, and 4 by cold knife or laser conization.

Histological results in the cone specimens. Diagnoses from the cone specimens of all 149 women were retrieved. Thirty-three (22\%) contained CIN1, 32 (21\%) CIN2, and 74 (49\%) CIN3. Four contained (3\%) adenocarcinoma in situ, two of which also contained CIN3. Six (4\%) cone specimens contained no CIN, leaving 143 women (96\% of 149) in the final
Table I. Conization results for 149 invited women ${ }^{\mathrm{a}}$ and 143 included women.

$\begin{array}{ll}\text { Characteristics } & \mathrm{n}(\%)\end{array}$

Histology results in cone specimens

of invited women $(n=149)$

$\mathrm{CIN}^{+} / \mathrm{AIS}$

CIN2

CIN1

No CIN ${ }^{b}$

Margin status of women included

in final analyses $(n=143)$

Free margins

Positive margins

No information about marginsc

2 (1.4)

CIN, cervical intraepithelial neoplasia; AIS, adenocarcinoma in situ; ${ }^{\mathrm{a}}$ mean age, 32.8 years, median 30 , range, 21-74. ${ }^{\mathrm{b}}$ Excluded from analyses; 'treated by cryotherapy.

analyses (Table I). Eighty-nine (62\%) cone specimens had free margins (complete excision according to the histological findings), and the remaining $52(36 \%)$ had positive margins (the diagnosis was not clarified due to uncertain resection margins (char/thermal artefact). In the two women (1\%) treated with cryotherapy, no information was available on margin status or histology, and instead the pre-treatment histological diagnoses (CIN1 and CIN2) were used.

Inclusion follow-up and subsequent visits. The inclusion visit consisted of a complete work-up, including pelvic exam, cytology testing, HPV DNA, and HPV E6/E7 mRNA testing. When indicated, colposcopy-directed biopsies were also taken. Women were divided in two groups based on when post-surgical HPV DNA and mRNA analysis were performed: 'early' (<12 months) and 'late' group (12 or more month after conization).

All 143 women had at least one additional follow-up visit after the inclusion visit (subsequent/final visit), consisting of Pap smear and, when clinically indicated, colposcopy-directed biopsies. Cytological results from these subsequent/final visits were available for 137 (96\% of 143) women and histological results for 30 (21\% of 143). The average follow-up time was 1,333 days (median 1,182, range 71-5,622 days), or 3.6 years.

Cytological and histological assessment. Cells from the ectoand endocervix were collected with an Ayres spatula and a cervical brush, smeared on a glass slide, and immediately fixed in $95 \%$ ethanol and air-dried for cytological examination (20). Cytological smears were classified according to the CIN classification of the Swedish Society for Clinical Cytology (21). For the purposes of this study, cytological results were re-classified using the Bethesda system, excluding koilocytosis without nuclear atypia from the low-grade squamous intraepithelial lesion (LSIL) group (22). 
Table II. Combined cytological/histological outcome during follow-up.

\begin{tabular}{lccc}
\hline Diagnosis & Inclusion visit N (\%) & Subsequent/last visit N (\%) & All follow-up visits N (\%) \\
\hline WNL & $114(79.7)$ & $124(86.7)$ & $110(76.9)$ \\
Low-grade disease $^{\mathrm{a}}$ & $20(14.0)$ & $12(8.4)$ & $25(17.5)$ \\
High-grade disease $^{\mathrm{b}}$ & $5(3.5)$ & $4(2.8)$ & $7(4.9)$ \\
Unsatisfactory $^{\mathrm{c}}$ & $4(2.8)$ & $3(2.1)$ & $1(0.7)$ \\
Total & 143 & 143 & 143 \\
\hline
\end{tabular}

WNL, without neoplastic lesions; CIN, cervical intraepithelial neoplasia; LSIL, low-grade squamous intraepithelial lesion; HSIL, high-grade squamous intraepithelial lesion. ${ }^{\mathrm{a}} \mathrm{CIN} 1 / \mathrm{LSIL}$; ${ }^{\mathrm{b}} \mathrm{CIN} 2 / \mathrm{HSIL}$; ${ }^{\mathrm{C}}$ istology was unsatisfactory for diagnosis.

When indicated by colposcopy, cervical biopsies were taken, preserved in formaldehyde and assessed by pathologists. In the absence of histology, the most severe cytological result (HSIL or worse, $\mathrm{HSIL}^{+}$) was used, whereas cytological results of atypical squamous cells of undetermined significance (ASCUS) and within normal limits (WNL) were considered normal.

For the purposes of this study, two combinations of cytological/histological results were defined: low-grade disease, including LSIL/CIN1, and high-grade disease including $\mathrm{HSIL}^{+} / \mathrm{CIN} 2$ or worse $\left(\mathrm{CIN} 2^{+}\right.$, i.e. CIN2, CIN3, carcinoma in situ and adenocarcinoma in situ).

HPV DNA analysis. Samples for HPV DNA testing were collected at the inclusion visit in the same manner as for cytology, and suspended in PreservCyt solution (ThinPrep ${ }^{\circledR}$, Hologic, Marlborough, MA, USA). DNA was extracted from the suspensions using the MagNA Pure LC robot (Roche Diagnostics, Basel, Switzerland) according to the manufacturer's instructions. HPV DNA detection and genotyping were carried out using the Linear Array (LA) HPV Genotyping Test (Roche Diagnostics). Briefly, HPV DNA was amplified by PCR using a pool of biotin-labeled primers that hybridize in the $\mathrm{L} 1$ region $(18,23,24)$. The $37 \mathrm{HPV}$ types included in the LA test were divided into three categories: HR, HPV16, $18,31,33,35,39,45,51,52,56,58$ and 59; probable HR (PHR), HPV26, 53, 66, 68, 73 and 82; and low-risk (LR) or undetermined-risk, HPV6, 11, 40, 42, 54, 55, 61, 62, 64, 67, 69, 70, 71, 72, 81, 83, 84, IS39 and CP6108 (25). For the purposes of this study, undetermined-risk and LR HPV types were combined.

HPV E6/E7 mRNA analysis by APTIMA HPV assay. Liquidbased samples used for HPV DNA analysis at the inclusion visit were retrieved from the archives and used for HPV E6/ E7 mRNA analysis. The PreservCyt sample was transferred to $2.9 \mathrm{ml}$ of buffered detergent solution, and a $400 \mu 1$ aliquot of the mixture was then tested by APTIMA HPV assay (Gene-Probe Inc., San Diego, CA, USA) according to the manufacturer's instructions.

The APTIMA HPV assay is a qualitative nucleic acid amplification test that detects the HPV E6/E7 mRNA of 14 HR HPV types $(16,18,31,33,35,39,45,51,52,56,58,59,66$ and 68 ), and has been validated for cervical specimens collected in PreservCyt solution. The test does not differentiate between HR HPV types, and is designed not to cross-react with the LR HPV types 6, 11, 42, 43, 44, or PHR HPV53 (26-28). The APTIMA HPV assay involves three main steps. An analyte cut-off (CO) of 1.00 was used in the assay for determining HPV interpretation. All laboratory analysis were performed by the Department of Virology, Karolinska Hospital.

Statistical methods. Data were analyzed with the software STATISTICA 6.1 (Statsoft Inc., Tulsa, OK, USA). Treatment failure was defined at two disease thresholds: low-grade disease or worse, or high-grade disease or worse detected during follow-up. Accuracy parameters of the prediction of treatment failure according to these two thresholds were computed, including sensitivity, specificity, positive predictive value (PPV) and NPV.

\section{Results}

Cytological and histological diagnoses during follow-up. Each woman in the study group (mean age, 31.3 years, median: 30 , range, 21-56) had at least two follow-up visits (one inclusion visit and one subsequent/final visit), and 20 women had three or more.

The combined cytological and histological results (when available) from the inclusion visit yielded 114 (79.7\%) WNL, $20(14 \%)$ women with low-grade disease, five $(3.5 \%)$ with high-grade disease, and four (2.8\%) with insufficient results. The results from the subsequent/final follow-up visit yielded 124 (86.7\%) WNL, 12 (8.4\%) women with low-grade disease, $4(2.8 \%)$ with high-grade disease, and $3(2.1 \%)$ with unsatisfactory samples.

When all follow-up visits were taken into account, a total of $32(22.4 \%)$ treatment failures (25 women with low-grade and 7 with high-grade disease) were identified among the 143 study women (Table II).

HR HPV DNA and HR HPV E6/E7 mRNA results at inclusion visit. A total of 33 (23\%) of the 143 study women were HR HPV DNA-positive at the inclusion visit. The most frequent HPV types detected were HPV52 (4.2\%), 33 and 56 (3.5\%) each. HPV16, 18, 51, 58 and 66 were equally frequent (2.8\%) each. Six women had multiple HR HPV infections (4 with a double, 1 with a triple and one with a quadruple infection). 
Table III. Discordant results for 26 women.

Histological/

Case no. HPV DNA HPV mRNA cytological outcome

\begin{tabular}{|c|c|c|c|}
\hline 25 & 66 & 0 & WNL \\
\hline 30 & $16,51,59$ & 0 & CIN2/HSIL \\
\hline 32 & 52 & 0 & CIN1/LSIL \\
\hline 35 & 18 & 0 & WNL \\
\hline 37 & 16 & 0 & WNL \\
\hline 40 & 66 & 0 & WNL \\
\hline 49 & 51,73 & 0 & WNL \\
\hline 57 & 33 & 0 & CIN1/LSIL \\
\hline 59 & 18 & 0 & WNL \\
\hline 71 & 66 & 0 & WNL \\
\hline 79 & 33 & 0 & CIN2/HSIL \\
\hline 81 & 82 & $0^{\mathrm{a}}$ & CIN1/LSIL \\
\hline 103 & 53 & $0^{\mathrm{a}}$ & CIN1/LSIL \\
\hline 104 & 31,66 & 0 & CIN1/LSIL \\
\hline 107 & 52 & 0 & WNL \\
\hline 108 & 66 & 0 & CIN2/HSIL \\
\hline 110 & 58 & 0 & WNL \\
\hline 115 & 58 & 0 & WNL \\
\hline 122 & 56 & 0 & WNL \\
\hline 131 & 51 & 0 & WNL \\
\hline 136 & 58 & 0 & WNL \\
\hline 138 & 16 & 0 & WNL \\
\hline 151 & 56 & 0 & CIN1/LSIL \\
\hline 18 & 0 & + & WNL \\
\hline 129 & 0 & + & WNL \\
\hline 164 & 0 & + & WNL \\
\hline
\end{tabular}

HPV L1 DNA and HPV E6/E7 mRNA by linear array and APTIMA HPV assay techniques, respectively. WNL, without neoplastic lesions; CIN, cervical intraepithelial neoplasia; LSIL, low-grade squamous intraepithelial lesion; HSIL, high-grade squamous

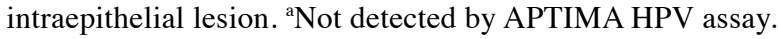

Only 13 (9.0\%) women were HR HPV E6/E7 mRNA-positive, less than half of that for HR HPV DNA. Cases that were HPV DNA-positive for types 33, 52, 56 (three cases each), and 18 (two cases), expressed mRNA more frequently than cases HPV DNA-positive for types 16, 31, 45 and 59, where E6/E7 mRNA was expressed in one case each.

Twenty-six discordant samples were found. Three HR HPV E6/E7 mRNA-positive women were HR HPV DNA-negative and had a cytological result of WNL. Among HR HPV E6/ E7 mRNA-negative women, two were HR HPV DNA-positive for types that are not included in the APTIMA HPV assay. Among the remaining $21 \mathrm{HPV}$ E6/E7 mRNA-negative, HPV DNA-positive women, 3 had high-grade, and 4 had low-grade disease (Table III).
Accuracy parameters of the prediction of treatment failure. Accuracy parameters of the prediction of treatment failure were calculated for two thresholds of disease: high-grade disease or worse and low-grade disease or worse.

Presence of HR HPV DNA at the inclusion visit predicted $100 \%$ (95\% CI 64.6-100) of residual/recurrent high-grade disease or worse, with a specificity of $80.9 \%(95 \% \mathrm{CI}$ 73.5-86.6). Cytology at the inclusion visit had a sensitivity of $85.7 \%$ (95\% CI 48.7-97.4), and a specificity of $87.5 \%$ (95\% CI 80.9-92.1) (Table IVA).

HPV E6/E7 mRNA was a poor predictor of treatment failure in the present study, with a sensitivity of $57.1 \%(95 \%$ CI 25.0-84.2). However, the test did have a high specificity (93.4\%; 95\% CI 87.9-96.5), PPV (30.8\%; 95\% CI 12.7-57.6) and NPV (97.7\%; 95\% CI 93.4-99.2). Margin status and presence of $\mathrm{CIN} 2^{+}$in the cone specimen were also poor predictors of treatment failure, with a sensitivity of $57.1 \%$ (95\% CI 25.0-84.2) and $71.4 \%$ (95\% CI 35.9-91.8), and a specificity of $64.2 \%$ (95\% CI 55.8-71.8) and 22.8\% (95\% CI 16.5-30.5), respectively (Table IVA).

Considering low-grade disease or worse as the threshold for treatment failure resulted in lower sensitivity and higher specificity values (Table IVB).

\section{Discussion}

To our knowledge, the present study is the first to compare the HPV E6/E7 mRNA of 14 HR HPV types and HPV DNA testing in the follow-up of women treated for CIN.

HPV infection is a necessary, but not sufficient, factor in the development of cervical neoplasia $(4,5,29)$. Certain HR HPV types $(16,18,45,31$ and 33$)$ are more frequently found in CIN2 ${ }^{+}$and SCC of the cervix (25). The oncogenic potential of these HPV types is due to the expression of the E6 and E7 oncoproteins and to their type-specific properties, such as affinity to tumor suppressors in the host cell (5). One would therefore expect HPV E6/E7 mRNA to be expressed in transforming cells, notably those infected with HR HPV. Such expression has been demonstrated in advancing grades of CIN using different mRNA tests, such as PreTect HPV Proofer (Norchip AS, Oslo, Norway), APTIMA HPV assay, and the real-time PCR technique (30-32), with improving sensitivity. We have previously reported on the expression of HR HPV mRNA using PreTect HPV Proofer compared to viral load and the tumor marker p16 $6^{\text {ink4a }}$ in different grades of cervical dysplasia (33). In that study, we observed a correlation between p16 $^{\text {ink4a }}$ and mRNA expression, but not viral load, nor increasing lesion severity. Probably not all HR HPV-infected cases have transcriptionally active E6/E7 expression, and absent mRNA may also represent regressing dysplasia as a consequence of E6/E7 expression being switched off (34).

Studies by Ratnam et al (31) and Dockter et al (35) reported a sensitivity of $91-95 \%$ for mRNA by APTIMA HPV assay to detect CIN2 ${ }^{+}$in women with abnormal cytology, whereas the specificity was between 55 and 43\%. Thus, our expectation was that an mRNA test targeting 14 HR HPV types would have a high sensitivity compared with HPV DNA testing, with a high specificity even after CIN treatment. In the present study, $23 \%$ of women harbored HR HPV DNA after treatment. Less than half of these women were also HR HPV E6/E7 
Table IV. Accuracy parameters of the prediction of treatment failure for high-grade disease or worse and low-grade disease or worse.

A, High-grade disease or worse

\begin{tabular}{|c|c|c|c|c|}
\hline Criteria & $\begin{array}{c}\text { Sensitivity \% } \\
(95 \% \mathrm{CI})\end{array}$ & $\begin{array}{l}\text { Specificity \% } \\
(95 \% \mathrm{CI})\end{array}$ & $\begin{array}{c}\text { PPV \% } \\
(95 \% \text { CI) }\end{array}$ & $\begin{array}{l}\text { NPV \% } \\
(95 \% \text { CI) }\end{array}$ \\
\hline High-risk HPV DNA & $100.0(64.6-100)$ & $80.9(73.5-86.6)$ & $21.2(10.7-37.8)$ & $100(96.6-100)$ \\
\hline Cytology at inclusion visit $\left(\text { ASCUS }^{+}\right)^{\mathrm{a}}$ & $85.7(48.7-97.4)$ & $87.5(80.9-92.1)$ & $26.1(12.6-46.5)$ & $99.2(95.4-99.9)$ \\
\hline High-risk HPV mRNA & $57.1(25.0-84.2)$ & $93.4(87.9-96.5)$ & $30.8(12.7-57.6)$ & $97.7(93.4-99.2)$ \\
\hline Margin status & $57.1(25.0-84.2)$ & $64.2(55.8-71.8)$ & $7.7(3.0-18.2)$ & $96.6(90.6-98.9)$ \\
\hline Cone containing $\mathrm{CIN} 2^{+}$ & $71.4(35.9-91.8)$ & $22.8(16.5-30.5)$ & $4.5(2.0-10.2)$ & $93.9(80.4-98.3)$ \\
\hline
\end{tabular}

B, Low-grade disease or worse

\begin{tabular}{lcccc}
\hline Criteria & $\begin{array}{c}\text { Sensitivity } \% \\
(95 \% \mathrm{CI})\end{array}$ & $\begin{array}{c}\text { Specificity } \% \\
(95 \% \mathrm{CI})\end{array}$ & $\begin{array}{c}\text { PPV \% } \\
(95 \% \mathrm{CI})\end{array}$ & $\begin{array}{c}\text { NPV \% } \\
(95 \% \mathrm{CI})\end{array}$ \\
\hline High risk HPV DNA & $53.3(34.3-71.7)$ & $84.4(76.2-90.6)$ & $48.5(30.8-66.5)$ & $86.8(78.8-92.6)$ \\
Cytology at inclusion visit (ASCUS $)^{\mathrm{a}}$ & $73.3(54.1-87.7)$ & $99.1(95.1-100)$ & $95.6(78.1-99.9)$ & $93.2(87.1-97.0)$ \\
High-risk HPV mRNA & $23.3(9.9-42.3)$ & $94.6(88.7-98)$ & $53.9(25.1-80.8)$ & $82.2(74.5-88.3)$ \\
Margin status & $36.7(19.9-56.1)$ & $64(54.3-72.9)$ & $21.6(11.3-35.3)$ & $78.9(69.0-86.8)$ \\
\hline
\end{tabular}

CIN, cervical intraepithelial neoplasia; HPV, human papillomavirus; PPV, positive predictive value; NPV, negative predictive value; CI, confidence interval; ASCUS, atypical squamous cells of undetermined significance. ${ }^{a}$ Cytological results that showed more than atypical squamous cells of ASCUS were considered abnormal.

mRNA-positive. HR HPV E6/E7 mRNA testing by APTIMA HPV assay had a low sensitivity (57.1\%) to predict residual/ recurrent high-grade disease, but a high specificity $(93.4 ; 95 \%$ CI 87.9-96.5) resulting in an NPV of $97.7 \%$. According to Verguts $e t a l$, sensitivity and NPV are more important than specificity and PPV in the follow-up management of women at risk of developing of cervical cancer compared to a screening setting (36). Among HPV E6/E7 mRNA-negative women in our study, three cases of high-grade disease were missed by the APTIMA HPV assay, despite the observed high NPV (as high as $97.7 \%$ ). We therefore concluded that the high NPV was misleading.

Negative HPV E6/E7 mRNA could have been due to the distribution of HR HPV types in archived samples. Indeed, prevalence of HPV16 and 18 was low, and HPV33, 52, and 56 were most frequent. Interestingly, these three HPV types were more frequently associated with HPV E6/E7 mRNA positivity than HPV16 and 18. False-negative HPV E6/E7 mRNA results are a possibility, although studies on the clinical performance of the APTIMA HPV assay indicate that mRNA remains stable and can be detected to the same extent as DNA $(27,35)$. mRNA quality may be another explanation, as samples were stored at room temperature, and the time between collection and testing was sometimes as much as 6-12 months.

Our study is the first to evaluate HPV E6/E7 mRNA expression after treatment by conization using the APTIMA HPV assay, which detects the E6/E7 mRNA of 14 HR HPV types. Only one previous study has compared HPV E6/E7
mRNA expression to HPV DNA detection in follow-up after CIN treatment, using PreTect HPV Proofer (37). The aforementioned study had a shorter follow-up period, but found a similarly low sensitivity and low PPV. This could have been due to false-negative results, since 8 of the 12 histologicallyverified $\mathrm{CIN}^{+}$in the study were HPV DNA-positive for an HPV type included in the mRNA assay. The authors concluded that a negative HPV mRNA result could not exclude the risk of malignant progression (37).

HPV DNA testing is increasingly used as a screening method, either alone or in conjunction with cytology. Its high sensitivity, ease and reproducibility make it very attractive as a first-round screening tool (Fröberg et al, unpublished data). Indeed, the high NPV of HPV DNA allows to safely referring women back to screening (Fröberg et al, unpublished data). However, HPV infections are often transient, especially in younger women, making overtreatment a serious problem.

In accordance with results from our previous study (18), HPV DNA testing in post-treatment follow-up identified all residual/recurrent high-grade CIN, and presence of HR HPV predicted $100 \%$ of high-grade disease with a specificity of $80.9 \%$. Indeed, HPV DNA-negative women in our study had only a negligible risk of treatment failure.

A summary of meta-analyses (15) concluded that a positive HPV DNA result is a better predictor of treatment failure than cytology or positive resection margins. However, based on our results, HPV E6/E7 mRNA testing by APTIMA HPV assay is not useful in the follow-up of women treated for CIN. 
In the future, one could envision a follow-up strategy that includes both HPV DNA and cytology. Women who are HPV DNA-negative at 6 and 24 months after treatment could be safely referred to the usual screening program, while HPV DNA-positive women should be followed annually until negative.

Careful surveillance of women treated for CIN is still required, as these women are at a higher risk of developing cervical cancer than the general population. Meta-analyses are needed to help establish an optimal follow-up strategy for women treated for CIN.

\section{Acknowledgements}

We thank Hamzah Safari for technical assistance and Trudy Perdrix-Thoma for editing assistance and English language review. This study was supported by the Swedish Cancer Foundation (070623, CAN 2007/1044), Karolinska Institutet Cancer Strategic Grants (5888/05-722), the Swedish Research Council (521-2008-2899), the Medical Research Council, and the Cancer Society in Stockholm, the Stockholm County Council, and Swedish Labour Market Insurance.

\section{References}

1. Arbyn M, Castellsagué X, de Sanjosé S, Bruni L, Saraiya M and Bray $\mathrm{F}$ and Ferlay J: Worldwide burden of cervical cancer in 2008. Ann Oncol 22: 2675-2686, 2011.

2. Hemminki K, Li X and Vaittinen P: Time trends in the incidence of cervical and other genital squamous cell carcinomas and adenocarcinomas in Sweden, 1958-1996. Eur J Obstet Gynecol Reprod Biol 101: 64-69, 2002.

3. Miller MG, Sung HY, Sawaya GF, Kearney KA, Kinney W and Hiatt RA: Screening interval and risk of invasive squamous cell cervical cancer. Obstet Gynecol 101: 29-37, 2003.

4. Walboomers JM, Jacobs MV, Manos MM, et al: Human papillomavirus is a necessary cause of invasive cervical cancer worldwide. J Pathol 189: 12-19, 1999.

5. zur Hausen H: Papillomaviruses and cancer: from basic studies to clinical application. Nat Rev Cancer 2: 342-350, 2002.

6. Vousden K: Interactions of human papillomavirus transforming proteins with the products of tumor suppressor genes. FASEB J 7: 872-879, 1993.

7. Livingstone LR, White A, Sprouse J, Livanos E, Jacks T and Tlsty TD: Altered cell cycle arrest and gene amplification potential accompany loss of wild-type p53. Cell 70: 923-935, 1992.

8. Peitsaro P, Johansson B and Syrjanen S: Integrated human papillomavirus type 16 is frequently found in cervical cancer precursors as demonstrated by a novel quantitative real-time PCR technique. J Clin Microbiol 40: 886-891, 2002.

9. Dowhanick JJ, McBride AA and Howley PM: Suppression of cellular proliferation by the papillomavirus E2 protein. J Virol 69: 7791-7799, 1995.

10. Nakagawa S, Yoshikawa H, Yasugi T, et al: Ubiquitous presence of E6 and E7 transcripts in human papillomavirus-positive cervical carcinomas regardless of its type. J Med Virol 62: 251-258, 2000

11. Cuschieri KS, Whitley MJ and Cubie HA: Human papillomavirus type specific DNA and RNA persistence - implications for cervical disease progression and monitoring. J Med Virol 73: 65-70, 2004.

12. Sotlar K, Selinka HC, Menton M, Kandolf R and Bultmann B: Detection of human papillomavirus type 16 E6/E7 oncogene transcripts in dysplastic and nondysplastic cervical scrapes by nested RT-PCR. Gynecol Oncol 69: 114-121, 1998.

13. Andersson S, Dillner L, Elfgren K, Mints M, Persson M and Rylander E: A comparison of the human papillomavirus test and Papanicolaou smear as a second screening method for women with minor cytological abnormalities. Acta Obstet Gynecol Scand 84: 996-1000, 2005.
14. Sotlar K, Diemer D, Stubner A, et al: Detection of high-risk human papillomavirus (HPV) E6 and E7 oncogene transcripts increases the specificity of the detection of a cervical intraepithelial neoplasia (CIN). Verh Dtsch Ges Pathol 89: 195-200, 2005 (In German).

15. Arbyn M, Sasieni P, Meijer CJ, Clavel C, Koliopoulos G and Dillner J: Chapter 9: clinical applications of HPV testing: a summary of meta-analyses. Vaccine 24 (Suppl 3): S3/78-89, 2006.

16. Zielinski GD, Bais AG, Helmerhorst TJ, et al: HPV testing and monitoring of women after treatment of CIN 3: review of the literature and meta-analysis. Obstet Gynecol Surv 59: 543-553, 2004.

17. Arbyn M, Paraskevaidis E, Martin-Hirsch P, Prendiville W and Dillner J: Clinical utility of HPV-DNA detection: triage of minor cervical lesions, follow-up of women treated for high-grade CIN: an update of pooled evidence. Gynecol Oncol 99 (3 Suppl 1): S7-S11, 2005.

18. Brismar S, Johansson B, Borjesson M, Arbyn M and Andersson S: Follow-up after treatment of cervical intraepithelial neoplasia by human papillomavirus genotyping. Am J Obstet Gynecol 201: 17 e1-8, 2009.

19. Mints M, Gaberi V and Andersson S: Miniconization procedure with C-LETZ conization electrode for treatment of cervical intraepithelial neoplasia: a Swedish study. Acta Obstet Gynecol Scand 85: 218-223, 2006

20. Arbyn M, Herbert A, Schenck U, et al: European guidelines for quality assurance in cervical cancer screening: recommendations for collecting samples for conventional and liquid-based cytology. Cytopathology 18: 133-139, 2007.

21. Richart RM: Cervical intraepithelial neoplasia. Pathol Annu 8: 301-328, 1973.

22. Solomon D, Davey D, Kurman R, et al: The 2001 Bethesda System: terminology for reporting results of cervical cytology. JAMA 287: 2114-2119, 2002.

23. Söderlund-Strand A, Rymark P, Andersson P, Dillner J and Dillner L: Comparison between the Hybrid Capture II test and a PCR-based human papillomavirus detection method for diagnosis and posttreatment follow-up of cervical intraepithelial neoplasia. J Clin Microbiol 43: 3260-3266, 2005.

24. Monsonego J, Pollini G, Evrard MJ, Sednaoui P, Monfort L, Zerat L and Syrjänen K: Detection of human papillomavirus genotypes among high-risk women: a comparison of hybrid capture and linear array tests. Sex Transm Dis 35: 521-527, 2008.

25. Muñoz N, Bosch FX, de Sanjosé S, et al: Epidemiologic classification of human papillomavirus types associated with cervical cancer. N Engl J Med 348: 518-527, 2003.

26. Getman D, Aiyer A, Dockter J, Giachetti C, Zhang F and Ginocchio CC: Efficiency of the APTIMA HPV assay for detection of HPV RNA and DNA targets. J Clin Virol 45 (Suppl 1): S49-S54, 2009.

27. Dockter J, Schroder A, Eaton B, Wang A, Sikhamsay N, Morales L and Giachetti C: Analytical characterization of the APTIMA HPV Assay. J Clin Virol 45 (Suppl 1): S39-S47, 2009.

28. Castle PE, Dockter J, Giachetti C, et al: A cross-sectional study of a prototype carcinogenic human papillomavirus E6/E7 messenger RNA assay for detection of cervical precancer and cancer. Clin Cancer Res 13: 2599-2605, 2007.

29. Bosch FX and Muñoz N: The viral etiology of cervical cancer Virus Res 89: 183-190, 2002.

30. Molden T, Kraus I, Karlsen F, Skomedal H, Nygard JF and Hagmar B: Comparison of human papillomavirus messenger RNA and DNA detection: a cross-sectional study of 4,136 women $>30$ years of age with a 2-year follow-up of high-grade squamous intraepithelial lesion. Cancer Epidemiol Biomarkers Prev 14: 367-372, 2005.

31. Ratnam S, Coutlee F, Fontaine D, et al: Aptima HPV E6/E7 mRNA test is as sensitive as Hybrid Capture 2 Assay but more specific at detecting cervical precancer and cancer. J Clin Microbiol 49: 557-564, 2011.

32. Andersson E, Karrberg C, Radberg T, et al: Type-specific human papillomavirus E6/E7 mRNA detection by real-time PCR improves identification of cervical neoplasia. J Clin Microbiol 49: 3794-3799, 2011

33. Andersson S, Hansson B, Norman I, et al: Expression of E6/E7 mRNA from 'high risk' human papillomavirus in relation to CIN grade, viral load and p16INK4a. Int J Oncol 29: 705-711, 2006. 
34. Nobbenhuis MA, Walboomers JM, Helmerhorst TJ, et al: Relation of human papillomavirus status to cervical lesions and consequences for cervical-cancer screening: a prospective study. Lancet 354: 20-25, 1999.

35. Dockter J, Schroder A, Hill C, Guzenski L, Monsonego J and Giachetti C: Clinical performance of the APTIMA HPV Assay for the detection of high-risk HPV and high-grade cervical lesions. J Clin Virol 45 (Suppl 1): S55-S61, 2009.
36. Verguts J, Bronselaer B, Donders G, Arbyn M, Van Eldere J, Drijkoningen $M$ and Poppe W: Prediction of recurrence after treatment for high-grade cervical intraepithelial neoplasia: the role of human papillomavirus testing and age at conisation. BJOG 113: 1303-1307, 2006.

37. Tropé A, Jonassen CM, Sjoborg KD, Nygard M, Dahl FA, Alfsen GC and Lie AK: Role of high-risk human papillomavirus (HPV) mRNA testing in the prediction of residual disease after conisation for high-grade cervical intraepithelial neoplasia. Gynecol Oncol 123: 257-262, 2011. 